\title{
Si-containing block copolymers for self-assembled nanolithography
}

\author{
C. A. Ross, ${ }^{\text {a) }}$ Y. S. Jung, V. P. Chuang, and F. llievski \\ Department of Materials Science and Engineering, Massachusetts Institute of Technology, Cambridge, \\ Massachusetts 02139 \\ J. K. W. Yang \\ Department of Electrical Engineering and Computer Science, Massachusetts Institute of Technology, \\ Cambridge, Massachusetts 02139 \\ I. Bita and E. L. Thomas \\ Department of Materials Science and Engineering, Massachusetts Institute of Technology, Cambridge, \\ Massachusetts 02139 \\ Henry I. Smith and K. K. Berggren \\ Department of Electrical Engineering and Computer Science, Massachusetts Institute of Technology, \\ Cambridge, Massachusetts 02139 \\ G. J. Vancso \\ MESA + Research Institute, University of Twente, P.O. Boc 217, 7500 Enschede, The Netherlands \\ J. Y. Cheng \\ IBM Almaden Research Center, San Jose, California
}

(Received 18 June 2008; accepted 18 August 2008; published 1 December 2008)

\begin{abstract}
Block copolymers can self-assemble to generate patterns with nanoscale periodicity, which may be useful in lithographic applications. Block copolymers in which one block is organic and the other contains Si are appealing for self-assembled lithography because of the high etch contrast between the blocks, the high etch resistance of the Si-containing block, and the high Flory-Huggins interaction parameter, which is expected to minimize line edge roughness. The locations and long range order of the microdomains can be controlled using shallow topographical features. Pattern generation from poly(styrene)-poly(ferrocenyldimethylsilane) and poly(styrene)-poly(dimethylsiloxane) block copolymers, and the subsequent pattern transfer into metal, oxide, and polymer films, is described. (0) 2008 American Vacuum Society. [DOI: 10.1116/1.2981079]
\end{abstract}

\section{INTRODUCTION}

The ability of block copolymers to self-assemble into periodic nanoscale structures makes them attractive for the lithographic patterning of nanoscale features ${ }^{1-7}$ such as patterned magnetic media. ${ }^{8,9}$ Moreover, the processing requirements for block copolymers (spin coating, annealing, reactive ion etching) are compatible with semiconductor processing techniques. The geometry of the self-assembled structures (e.g., arrays of spheres, cylinders, or lamellae) and their length scales can be controlled via the chain lengths of the blocks from which the polymer is composed. The long range ordering and the registration of the features can be controlled by using chemical ${ }^{10-13}$ or topographical ${ }^{14-21}$ patterns on the substrates, and placement to within 2-3 nm of a reference feature has been demonstrated. ${ }^{22}$ These characteristics make block copolymer lithography a potentially cost-effective method for the formation of dense arrays of nanoscale features.

The majority of block copolymer patterning work has been based on poly(styrene)-poly(methylmethacrylate) (PSPMMA) diblock copolymers. In this system the PMMA is easily removed, leaving the PS features on the substrate. However, PS is a poor etch mask, which limits subse-

a)Electronic mail: caross@mit.edu quent pattern transfer processes. By using a $\mathrm{Si}_{-}$or $(\mathrm{Si}+\mathrm{Fe})$-containing organometallic material as one block of the polymer, a more robust etch mask is formed, which enables the formation of high aspect ratio features and the use of subtractive processes for pattern transfer.

In this article we describe pattern formation and pattern transfer from two $\mathrm{Si}-$ or $(\mathrm{Si}+\mathrm{Fe})$-containing diblock copolymers, poly(styrene)-poly(ferrocenyldimethylsilane) (Refs. 23 and 24) (PS-PFS, synthesized at the University of Twente) and poly(styrene)-poly(dimethylsiloxane) (Ref. 25) (PSPDMS), synthesized by Polymer Source Inc. In both cases, after annealing to promote microphase separation of the blocks, the PS is removed with oxygen reactive ion etching, leaving oxidized PFS or PDMS features that can serve as masks for pattern transfer. We describe, in particular, the templating of the microdomains using topographical features consisting of shallow trenches or pits, or arrays of pillars, and discuss the effect of the commensurability between the block copolymer period and the size of the template. We describe the pattern transfer processes and the properties of the resulting structures, with examples of the formation of magnetic "dot" arrays for perpendicular patterned media and magnetic rings for magnetic random access memories or magnetic logic. 


\section{EXPERIMENTAL METHODS}

Topographical templates were made on oxidized silicon substrates. Patterns consisting of parallel lines or arrays of circular pits were defined in a trilayer resist stack by interference lithography over large areas $\left(\mathrm{a}\right.$ few $\mathrm{cm}^{2}$ ), or by electron-beam lithography, and transferred into the oxide layer using a series of reactive ion etch (RIE) steps. The thicknesses of the block copolymer layers were chosen such that, after annealing, the polymer filled the trenches leaving little or no material on the mesas. Pillars with diameters of $\sim 10 \mathrm{~nm}$ were made in hydrogen silsesquioxane (HSQ) resist using electron-beam lithography directly.

The PS-PFS, which contains $<\sim 20$ vol \% PFS, is spin coated then annealed at $140-180{ }^{\circ} \mathrm{C}$ to form a monolayer of close-packed PFS spheres within a PS matrix. The sphere row spacing is $25-50 \mathrm{~nm}$ depending on the molecular mass of the block copolymer. Molecular masses of 33-10, 47-15, $69-21$, and $91-21 \mathrm{~kg} / \mathrm{mol}$ PS-PFS were used, referred to as $33 / 10,47 / 15,69 / 21$, and $91 / 21$ respectively. After thermal annealing, typically at $180^{\circ} \mathrm{C}$ for several hours, the PS was removed with an oxygen RIE or with ozonolysis to leave an array of partly oxidized PFS spheres. ${ }^{17,26}$

One PS-PDMS block copolymer $(45.5 \mathrm{~kg} / \mathrm{mol}$ total molecular mass) contained 33.5 vol \% PDMS and formed an array of parallel PDMS cylinders with $34 \mathrm{~nm}$ period in a PS matrix on annealing. A second PS-PDMS block copolymer $(51.5 \mathrm{~kg} / \mathrm{mol}$ total molecular mass, $16.5 \mathrm{vol} \%$ PDMS $)$ formed an array of close-packed PDMS spheres with centerto-center spacing of $38 \mathrm{~nm}$ in a PS matrix on annealing. After spin coating, the PS-PDMS was solvent annealed at room temperature in an ambient of toluene vapor or annealed at $170-200{ }^{\circ} \mathrm{C}$, for several hours. PDMS has a much lower surface energy than PS and forms a surface layer at the polymer-air interface and also wets the silica surface. The PDMS surface layer was removed using a short $\mathrm{CF}_{4}$ RIE, then the PS was removed with $\mathrm{O}_{2}$ RIE to expose the PDMS cylinders. $^{25}$

The etched block copolymer morphologies were imaged by scanning electron microscopy, after metal coating. Pattern transfer into a number of materials was accomplished using sequences of reactive ion etching and ion beam etching processes.

\section{RESULTS AND DISCUSSION}

\section{A. Block copolymer selection}

The majority of block copolymer lithography research has been performed on PS-PMMA. This material is available in a range of molecular weights and volume fractions, and its processing techniques are well established. For example, PMMA can be selectively removed by degradation using UV light and dissolution by acetic acid or by etching using an oxygen, argon or $\mathrm{CF}_{4}$ plasma. However, the PS block which remains has poor thermal stability, with a glass transition temperature of approximately $100{ }^{\circ} \mathrm{C}$. PS structures have been used for additive patterning, such as electrodeposition ${ }^{27}$ or lift-off, ${ }^{28}$ but subtractive pattern transfer is more difficult because the PS is easily attacked by, for example, an oxygen plasma. Subtractive pattern transfer is instead accomplished by making a hard mask of a more robust material such as spin-on glass by an additive process, then using the hard mask to pattern an underlying film. ${ }^{15} \mathrm{Si}$ or $(\mathrm{Si}+\mathrm{Fe})$ containing block copolymers are advantageous because there is a high etch selectivity between the organometallic block and the organic block. ${ }^{29}$ For example, in PS-PDMS or PSPFS, the PS is removed by an oxygen plasma which also partly oxidizes the Si-containing block, leaving a material with properties similar to silica. The oxidized organometallic domains are sufficiently robust for subtractive pattern transfer using RIE or ion beam etching.

A second important consideration in selecting a block copolymer for self-assembled lithography is the value of the Flory-Huggins $\chi$ parameter, which describes the thermodynamic tendency of the block copolymer to microphase segregate. Microphase segregation occurs above a certain value of $\chi N$, approximately $10,{ }^{30}$ where $N$ is the number of monomer repeats in the chain. Materials with a higher $\chi$ can microphase segregate at a smaller chain length, yielding patterns with smaller period (in the strong segregation limit, the pattern period is given by $a N^{2 / 3} \chi^{1 / 6}$, where $a$ is the segment length). Additionally, the width of the interface between the two blocks is given by $a \chi^{-1 / 2},{ }^{30}$ implying that a block copolymer with higher $\chi$ will have sharper interfaces between the blocks, facilitating a smaller edge roughness in the features. The $\chi$ parameters at room temperature are 0.04-0.06 for PS-PMMA, ${ }^{31} 0.08$ for PS-PFS, ${ }^{32}$ and 0.26 for PS-PDMS ${ }^{33}$ suggesting that PS-PDMS can achieve about half the periodicity of PS-PMMA. The high $\chi$ of PS-PDMS has made it particularly attractive for the generation of welldefined patterns with long correlation lengths. Offsetting these advantages is the relative difficulty of obtaining materials and the scarcity of information about these block copolymers.

\section{B. Pattern generation from spherical-morphology PS-PFS}

Block copolymer films spin coated on a smooth substrate generate patterns with good short range order, but for many device applications, precise long range order is required. In our work, to impose long range order, topographical templates were used with depths of $30-50 \mathrm{~nm}$, the approximate thickness of a film containing a monolayer of spherical domains.

We examined, in particular, the behavior of PS-PFS $33 / 10$ when the size of the template is a few times the period of the block copolymer. In this regime, commensurability between the block copolymer and the template is important. Experimentally, the block copolymer adjusts its period in order to fit inside the template. This is most clearly revealed in a series of measurements on grooves of different widths. ${ }^{17}$ An example is shown in Fig. 1(a). For any value of groove width between about $d_{0}$ and $12 d_{0}$, where $d_{0}$ is the spacing between rows of spherical microdomains in the close-packed array, the block copolymer microdomains form a well- 


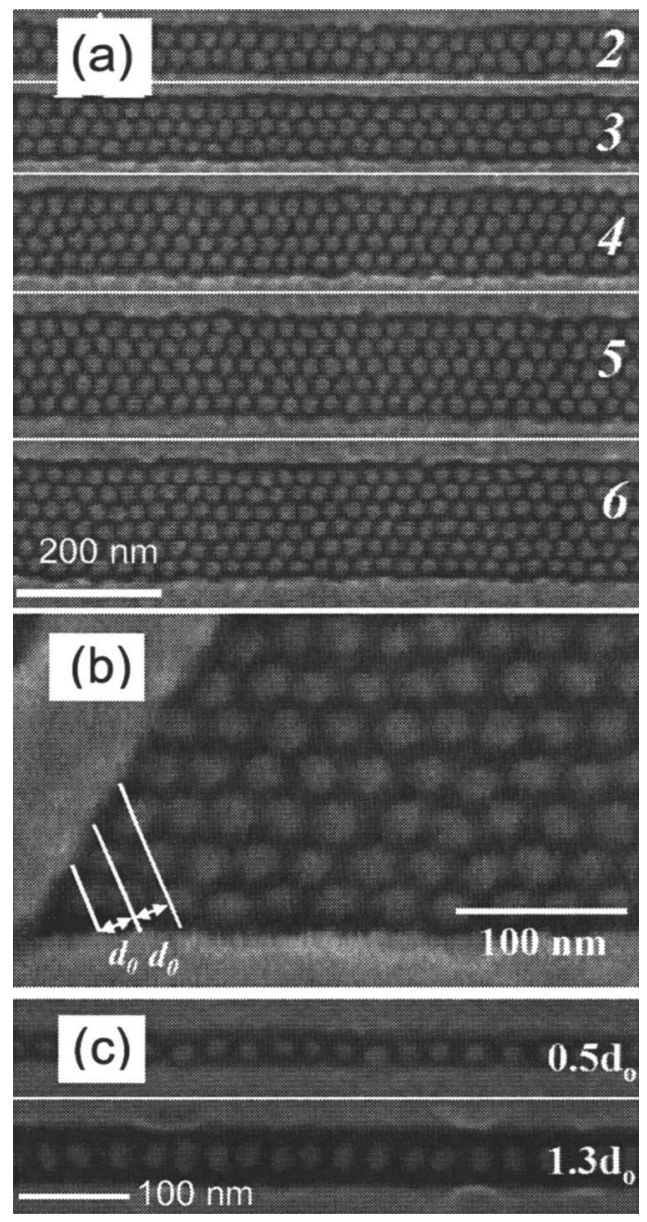

FIG. 1. Self-assembled PS-PFS 33/10 patterns. (a) In grooves of different widths, a composite image (from Ref. 17), (b) in a $60^{\circ}$ angle (from Ref. 22), (c) in narrow grooves, showing ellipsoidal distortion of the PFS microdomains (from Ref. 34). In each case the PS matrix has been selectively etched.

ordered close-packed array within the groove, with a spacing that expands or contracts to fit within the template. If the groove width is in the range of $(M-1 / 2) d_{0}$ to $(M+1 / 2) d_{0}$, where $M$ is an integer, $M$ rows of spherical domains form, i.e., there is always an integer number of rows. However, there is an overlap between the number of rows, for example, at a groove width near $(M+1 / 2) d_{0}$, either $M$ or $M+1$ rows may form. This behavior was understood in terms of the free energy of the array of microdomains, which increases as the array is strained compared to its equilibrium row spacing $d_{0}$.

The elastic compliance of the microdomain arrays exhibited in this experiment is critical in lithographic applications because it allows the block copolymer microdomain array to conform to an incommensurate or imperfect template, easing the fabrication requirements on the template. For example, if the template has edge roughness, the row of microdomains closest to the edge has positions that correlate well with the roughness of the template. However, as we move away from the edge, the correlation is lost, so that the microdomain array has better short range order. The array therefore demonstrates a "self-healing" effect in which perturbations of domain positions are damped out, and the array is tolerant to defects in the template. ${ }^{22}$ Figure 1(b) shows how spherical domains are templated by a sharp angle, such that the location of the corner domain is determined to within a few nanometers.

Commensurability effects are also observed in spherical microdomain arrays within narrow grooves, with widths of $(0.5-1.5) d_{0} \cdot{ }^{34}$ In these very narrow templates, only one row of spherical microdomains can form, but the domains distort into ellipsoids in order to fit within the template. This allows rows of elliptical structures to be made with controlled aspect ratio, as shown in Fig. 1(c).

PS-PFS of larger molecular mass also showed similar templating behavior in grooves. However, the ordering of the patterns, and the monodispersity of the microdomains, was considerably poorer. This is attributed to the lower diffusivity in higher molecular weight polymers, which impedes the formation of an equilibrium structure. The lower diffusivity for higher molecular weights may impose an upper limit on pattern periodicity in block copolymer self-assembly.

\section{Pattern generation from cylindrical- and spherical-morphology PS-PDMS}

PS-PDMS is attractive because of its high $\chi$ parameter and the high etch selectivity between the blocks. However, the PDMS block segregates strongly to the film surface and to the surface of the substrate. A short $\mathrm{CF}_{4}$ etch process is therefore necessary for the removal of the surface layer prior to $\mathrm{O}_{2}$-etching the PS matrix to reveal the PDMS microdomains. ${ }^{25}$ PS-PDMS was most conveniently annealed in solvent vapor, although thermal annealing at $170-200{ }^{\circ} \mathrm{C}$ is also effective in promoting microphase segregation. We describe first the behavior of cylindrical morphology PSDMS. In thin films the cylinders could be oriented either parallel or perpendicular to the edges of a topographical template depending on the annealing conditions and substrate geometry. This phenomenon is believed to be caused by flow-induced ordering of the cylinders. Longer anneals, higher vapor pressure, or the use of a substrate geometry with smaller mesas between the substrate grooves promote cylinder orientation parallel to the grooves. Figure 2 shows a plan view and tilted view of an array of PDMS cylinders aranged parallel to the template edge. The etched cylinders had a line edge roughness of $4 \mathrm{~nm}$, estimated from the electron micrograph. Figure 3(a) shows the cylinders arranged perpendicular to the template edge. ${ }^{25}$

In circular pits, the cylinders were curved to form concentric ring patterns with spacing governed by the pit diameter. ${ }^{35}$ Figure 4 shows concentric rings formed in templates of different widths. As with the ordering of PFS spherical domains, the number of concentric PDMS cylinders within a circular pit increases stepwise as the pit diameter increased. The spacing of the outer cylinders is similar to the equilibrium spacing, but inner cylinders are more closely spaced as a result of the contribution of the bending energy to the total energy of the system. This method allows precise control of circular or other concentric geometries using a template that is coarser than the linewidth of the patterns. 

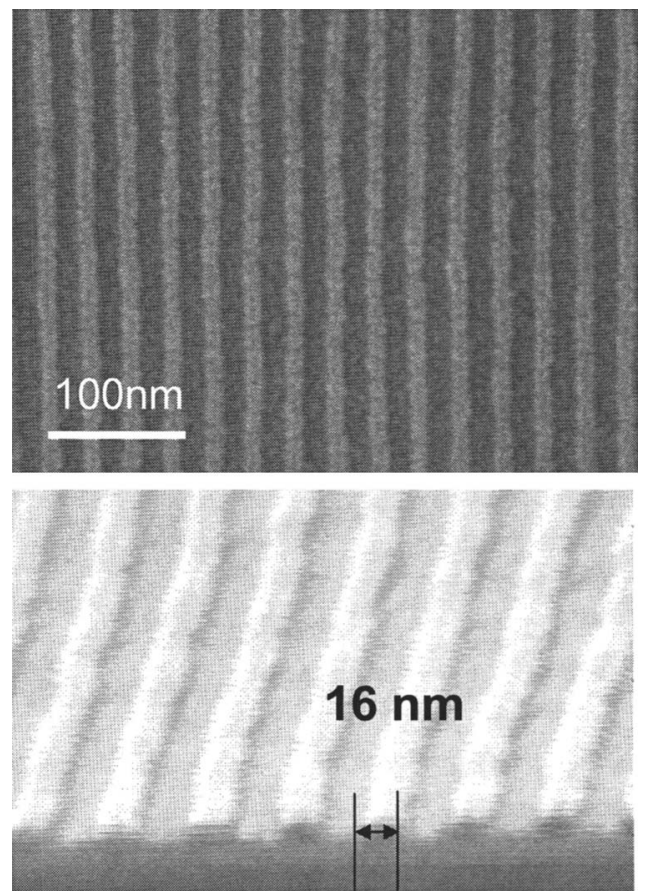

FIG. 2. Plan and side views of a section of a $32 \mathrm{~nm}$ period PDMS cylinder array ordered within a $875 \mathrm{~nm}$ wide shallow silica groove (groove edges are not shown). The PS matrix and PDMS surface layer have been etched.

In the work described above, block copolymers have been templated using shallow trenches or grooves. An alternative approach is to use a dilute array of posts to template of sphere array. ${ }^{36}$ The posts substitute for domains, pinning the location and orientation of the array, as illustrated for PDMS spheres in a PS-PDMS block copolymer. This enables the frequency of the template pattern to be multiplied by the
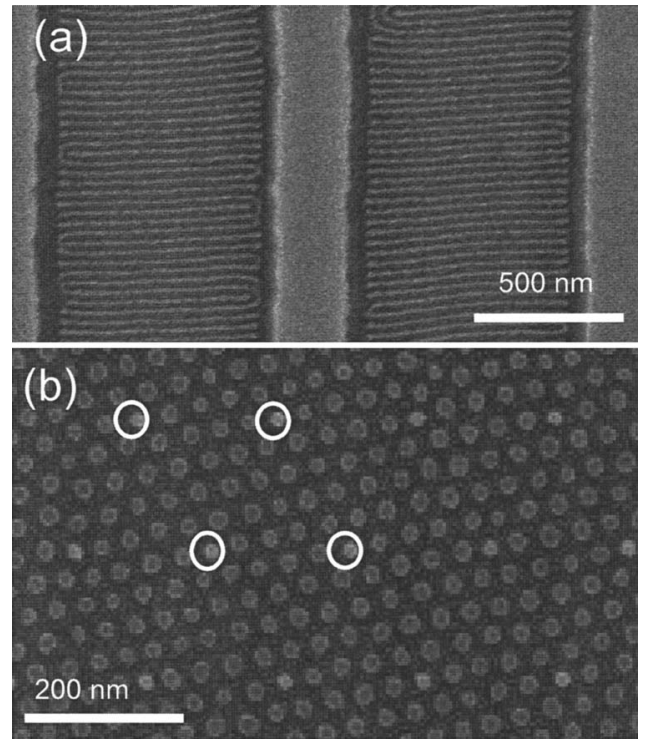

FIG. 3. (a) Plan view of $32 \mathrm{~nm}$ period PDMS cylinders oriented perpendicular to the edges of the template (from Ref. 25). (b) $38 \mathrm{~nm}$ period PDMS sphere array templated by a sparse array of HSQ pillars, some of which are circled (from Ref. 36). The PS matrix and PDMS surface layer have been etched.
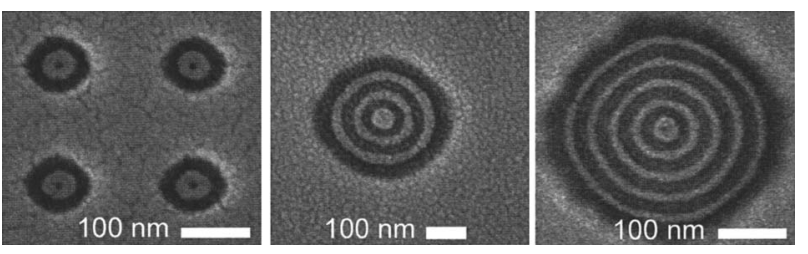

FIG. 4. PDMS cylinders confined within circular pits curve to follow the pit edges. The number of concentric rings is governed by the template diameter. The central feature can be a PS dot (dark) or a PDMS dot (white, middle image). The PS matrix and PDMS surface layer have been removed. From Ref. 35 .

self-assembly process. For example, in Fig. 3(b), the period of the post array is $\sqrt{21}$ times that of the block copolymer, and one unit cell of the post lattice contains 21 block copolymer unit cells. Moreover, the posts are incorporated into the array, and if designed appropriately, they become indistinguishable from the block copolymer domains on subsequent processing. For example, if the posts are made from HSQ, they behave similarly to the oxidized PDMS domains during etching processes. The templating is insensitive to the exact size and shape of the posts, relaxing the requirements on the patterning method used to generate the posts.

\section{Pattern transfer into metal, oxide, and polymer films}

Block copolymer patterns were transferred into various materials, including magnetic and nonmagnetic metals, silica, silicon, antireflective coatings, and conductive organic materials, using etching or lift-off processing. Transfer into metal films, such as magnetic films or multilayers, could be achieved by coating the magnetic metal (e.g., $\mathrm{Co}, \mathrm{NiFe}$, and $\mathrm{CoCrPt} / \mathrm{Ti}$ and $\mathrm{CoFe} / \mathrm{Cu} / \mathrm{NiFe}$ multilayers) with tungsten, then silica, then a PS-PFS block copolymer. ${ }^{37-39}$ The pattern of the PFS domains was transferred into silica using a $\mathrm{CHF}_{3}$ $\mathrm{RIE}$, then into $\mathrm{W}$ using $\mathrm{CF}_{4}+\mathrm{O}_{2} \mathrm{RIE}$, then into the Co by $\mathrm{Ne}^{+}$ion beam etching. An example is shown in Fig. 5(a) for a Co film patterned using PS-PFS 91/21, with $56 \mathrm{~nm}$ period. The substrate surface was flat, and in the absence of a template, the dot array has short range close packing, but no long range order. $\mathrm{CoCrPt} / \mathrm{Ti}$ was also patterned, using PS-PFS $47 / 15$ and 69/21, and in this case the magnetic "dots" show a strong perpendicular anisotropy due to the out-of-plane $c$-axis orientation of the Co-alloy induced by the Ti. ${ }^{39}$ The dots reverse their magnetization in a coherent manner, and interactions between neighbors are weak compared to the coercivity, making this a possible prototype for patterned recording media.

One issue with this pattern transfer method is that, if the block copolymer has been templated using topographic features, the pattern of the template is transferred into the underlying film at the same time as the block copolymer domain pattern. This can be avoided by using an etchable template such as a patterned polymer film. The template material must maintain its shape in the presence of the solvents used in spin coating or annealing of the polymer, yet be easily removed after the block copolymer has ordered. A 

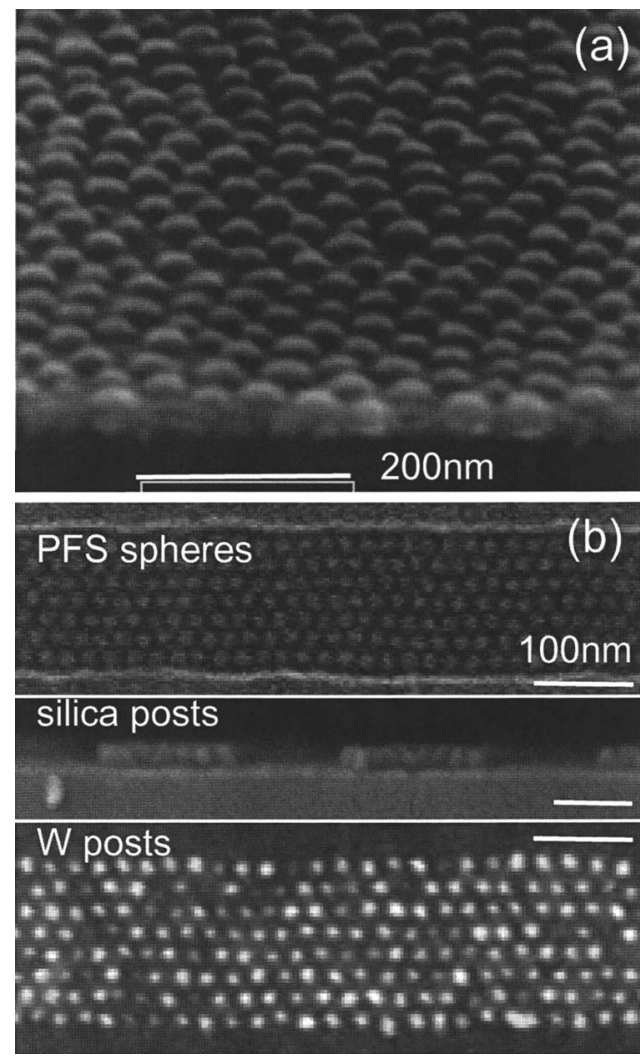

FIG. 5. (a) Co dot array with $56 \mathrm{~nm}$ period made by etching a Co film using PS-PFS $91 / 21$ as a mask. (b) Upper panel: PS-PFS 47/15 self-assembled in a template made from ARC. Middle panel: pattern transferred into silica, after removal of the ARC. Lower panel: pattern transferred into W.

BARLi antireflective coating (ARC) was found to be suitable. Figure 5(b) shows PS-PFS templated using stripes of $\mathrm{ARC}$, and the resulting pattern of ordered PFS dots on a smooth substrate after the ARC has been removed. On transferring this pattern into a $\mathrm{Cr}$ layer, an array of ordered $\mathrm{Cr}$ dots is formed, separated by spaces corresponding to the locations of the ARC templates.

Line and ring patterns of PDMS cylindrical microdomains with period of $32 \mathrm{~nm}$ have been transferred into several materials, including metals, oxide, and silica. An example of patterned concentric Co rings is shown in Fig. 6(a). Each structure consists of two concentric Co rings with a narrow gap between them. In this case the image contrast is reversed, i.e., the Co rings correspond to the location of the PS majority block, while the gap between the Co rings corresponds to the PDMS ring location. Such ring structures may be useful in a range of applications, including lasers, ${ }^{40}$ transistors, ${ }^{41}$ magnetic memories, ${ }^{42,43}$ sensors, ${ }^{44,45}$ or logic devices. ${ }^{46}$ Ring-shaped multilayer magnetic devices, in particular, have been studied extensively because they exhibit a range of controllable magnetization configurations enabling logic or multibit memory function. ${ }^{42,43,46}$

Figure 6(b) shows an array of parallel wires etched into a film of poly(3,4-ethylenedioxythiophane):poly(styrenesulfonate) (PEDOT:PSS), a conductive polymer, using an $\mathrm{O}_{2}$ RIE. ${ }^{47}$ Electrical measurements confirm that the conducting

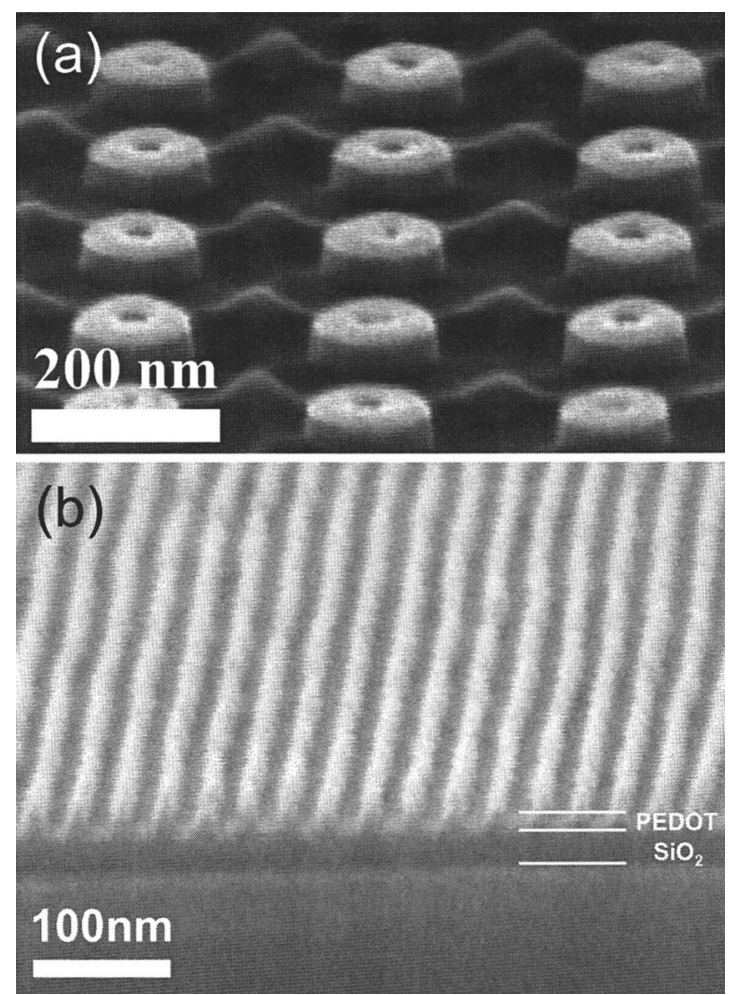

FIG. 6. (a) Array of Co rings. Each ring consists of two concentric rings, closely spaced. (From Ref. 35). (b) Array of $32 \mathrm{~nm}$ period lines etched into a PEDOT thin film (Ref. 47).

properties of the organic film are not degraded by the patterning process. These structures may have applications in gas sensors and other nanowire devices.

\section{CONCLUSIONS}

This article illustrates the use of block copolymers with a $\mathrm{Si}$ - or $(\mathrm{Si}+\mathrm{Fe})$-containing block for self-assembled lithography. These block copolymers are useful in this application because they have a high etch selectivity between the two blocks, and the organometallic domains that remain after removal of the organic block are robust masks for subtractive pattern transfer. In addition, these block copolymers often have high Flory-Huggins $\chi$ parameters, which is advantageous in terms of creating small-period arrays and in obtaining smooth interfaces between the microdomains.

The positions of microdomains can be templated using shallow topographical features such as trenches or pits or small pillars which "substitute" for one microdomain in the array. This allows well-controlled periodic or aperiodic ${ }^{17}$ patterns to be obtained, for example, close-packed arrays of dots, parallel lines, and concentric rings, by using a template with period that is an order of magnitude or more larger than the period of the block copolymer. The compliance of the block copolymer film allows the array of microdomains to conform to the template, making the pattern generation tolerant of edge roughness or incommensurability of the template dimensions. 
Block copolymer patterns have been transferred into a variety of materials, including metal films, oxide, silicon, and other polymers. This opens the possibility of low cost fabrication of devices with nanoscale features. The exploration of these materials has just begun, and there are several exciting paths forward, including the use of organometalliccontaining triblock copolymers such as poly(styrene)poly(ferrocenyldimethylsilane)-poly(2-vinylpyridine) (Ref. 48) to obtain a wider range of geometries, the addition of $\mathrm{Si}$ compounds to block copolymers to make more complex blends with high etch selectivity [e.g., the addition of organosilicate to the poly(ethylene oxide) (PEO) microdomains of a PS-PEO block copolymer], ${ }^{49}$ the use of materials which microphase separate at room temperature, ${ }^{21}$ and the use of Si-containing block copolymers with additional optical or chemical functionality.

\section{ACKNOWLEDGMENTS}

The authors gratefully acknowledge the support of the Semiconductor Research Corporation, the Singapore-MIT Alliance, and the National Science Foundation.

${ }^{1}$ G. Krausch and R. Magerle, Adv. Mater. (Weinheim, Ger.) 14, 1579 (2002).

${ }^{2}$ I. W. Hamley, Nanotechnology 14, R39 (2003).

${ }^{3}$ C. Park, J. Yoon, and E. L. Thomas, Polymer 44, 6725 (2003).

${ }^{4}$ R. A. Segalman, Mater. Sci. Eng., R 48, 191 (2005).

${ }^{5}$ J. Y. Cheng, C. A. Ross, E. L. Thomas, and H. I. Smith, Adv. Mater. (Weinheim, Ger.) 18, 2505 (2006).

${ }^{6}$ S. B. Darling, Prog. Polym. Sci. 32, 1152 (2007).

${ }^{7}$ C. T. Black et al., IBM J. Res. Dev. 51, 605 (2007).

${ }^{8}$ B. D. Terris and T. Thomson, J. Phys. D 38, R199 (2005).

${ }^{9}$ C. A. Ross, Annu. Rev. Mater. Sci. 31, 203 (2001).

${ }^{10}$ L. Rockford, Y. Liu, P. Mansky, T. P. Russell, M. Yoon, and S. G. J. Mochrie, Phys. Rev. Lett. 82, 2602 (1999).

${ }^{11}$ S. O. Kim, H. H. Solak, M. P. Stoykovich, N. J. Ferrier, J. J. de Pablo, and P. F. Nealey, Nature (London) 424, 411 (2003).

${ }^{12}$ E. W. Edwards, M. F. Montague, H. H. Solak, C. J. Hawker, and P. F. Nealey, Adv. Mater. (Weinheim, Ger.) 16, 1315 (2004).

${ }^{13}$ M. P. Stoykovich, M. Muller, S. O. Kim, H. H. Solak, E. W. Edwards, J. J. de Pablo, and P. F. Nealey, Science 308, 1442 (2005).

${ }^{14}$ R. A. Segalman, H. Yokoyama, and E. J. Kramer, Adv. Mater. (Weinheim, Ger.) 13, 1152 (2001).

${ }^{15}$ K. Naito, H. Hieda, M. Sakurai, Y. Kamata, and K. Asakawa, IEEE Trans. Magn. 38, 1949 (2002).

${ }^{16}$ C. T. Black and O. Bezencenet, IEEE Trans. Nanotechnol. 3, 412 (2004).

${ }^{17}$ J. Y. Cheng, A. M. Mayes, and C. A. Ross, Nat. Mater. 3, 823 (2004).
${ }^{18}$ D. Sundrani, S. B. Darling, and S. J. Sibener, Nano Lett. 4, 273 (2004).

${ }^{19}$ S. Xiao, X. Yang, E. W. Edward, Y. La, and P. F. Nealey, Nanotechnology 16, S324 (2005).

${ }^{20}$ R. Ruiz, R. L. Sandstrom, and C. T. Black, Adv. Mater. (Weinheim, Ger.) 19, 587 (2007).

${ }^{21}$ M. Roerdink, M. A. Hempenius, U. Gunst, H. F. Arlinghaus, and G. J. Vancso, Small 3, 1415 (2007).

${ }^{22}$ J. Y. Cheng, F. L. Zhang, H. I. Smith, G. J. Vancso, and C. A. Ross, Adv. Mater. (Weinheim, Ger.) 18, 597 (2006).

${ }^{23}$ Y. Ni, R. Rulkens, and I. Manners, J. Am. Chem. Soc. 118, 4102 (1996).

${ }^{24}$ R. G. H. Lammertink, M. A. Hempenius, J. G. Vancso, K. Shin, M. H. Rafaelovich, and J. Sokolov, Macromolecules 34, 942 (2001).

${ }^{25}$ Y. S. Jung and C. A. Ross, Nano Lett. 7, 2046 (2007).

${ }^{26}$ V. Z.-H. Chan et al., Science 286, 1716 (1999).

${ }^{27}$ T. Thurn-Albrecht et al., Science 290, 2126 (2000).

${ }^{28}$ S. Xiao, X. Yang, E. W. Edward, Y. La, and P. F. Nealey, Nanotechnology 16, S324 (2005).

${ }^{29}$ I. Korczagin, R. G. H. Lammertink, M. A. Hempenius, S. Golze, and G. J. Vancso, Adv. Polym. Sci. 200, 91 (2006).

${ }^{30}$ F. S. Bates and G. H. Fredrickson, Annu. Rev. Phys. Chem. 41, 525 (1990).

${ }^{31}$ T. P. Russell, R. P. Hjelm, and P. A. Seeger, Macromolecules 23, 890 (1990).

${ }^{32}$ H. B. Eitouni, N. P. Balsara, H. Hahn, J. A. Pople, and M. A. Hempenius, Macromolecules 35, 7765 (2002).

${ }^{33}$ T. Nose, Polymer 36, 2243 (1995)

${ }^{34}$ J. Y. Cheng, F. Zhang, A. M. Mayes, and C. A. Ross, Nano Lett. 6, 2099 (2006).

${ }^{35}$ Y. S. Jung, W. Jung, and C. A. Ross, Nano Lett. 8, 2975 (2008).

${ }^{36}$ I. Bita, J. K. W. Yang, Y. S. Jung, C. A. Ross, E. L. Thomas, and K. K. Berggren, Science 431, 939 (2008).

${ }^{37}$ J. Y. Cheng, C. A. Ross, V. Z. H. Chan, E. L. Thomas, R. G. H. Lammertink, and G. J. Vancso, Adv. Mater. (Weinheim, Ger.) 13, 1174 (2001).

${ }^{38}$ J. Y. Cheng, W. Jung, and C. A. Ross, Phys. Rev. B 70, 064417 (2004).

${ }^{39}$ F. Ilievski, C. A. Ross, and G. J. Vancso, J. Appl. Phys. 103, 07C520 (2008).

${ }^{40}$ M. T. Hill et al., Nature (London) 432, 206 (2004).

${ }^{41}$ H. Watanabe, C. Manabe, T. Shigematsu, and M. Shimizu, Appl. Phys. Lett. 78, 2928 (2001).

${ }^{42}$ J. G. Zhu, Y. F. Zheng, and G. A. Prinz, J. Appl. Phys. 87, 6668 (2000).

${ }^{43}$ F. J. Castaño, D. Morecroft, W. Jung, and C. A. Ross, Phys. Rev. Lett. 95, 137201 (2005).

${ }^{44}$ M. M. Miller, G. A. Prinz, S. F. Cheng, and S. Bounnak, Appl. Phys. Lett. 81, 2211 (2002).

${ }^{45}$ J. Llandro, T. J. Hayward, D. Morecroft, J. A. C. Bland, F. J. Castaño, I. A. Colin, and C. A. Ross, Appl. Phys. Lett. 91, 203904 (2007).

${ }^{46}$ F. J. Castaño, B. G. Ng, I. A. Colin, D. Morecroft, W. Jung, and C. A. Ross, J. Phys. D 41, 132005 (2008).

${ }^{47}$ Y. S. Jung, W. C. Jung, and H. L. Tuller, C. A. Ross (unpublished)

${ }^{48}$ I. A. Manners (unpublished).

${ }^{49}$ V. P. Chuang, W. Jung, C. A. Ross, J. Y. Cheng, Q.-H. Park, and H.-C. Kim, J. Appl. Phys. 103, 074307 (2008). 